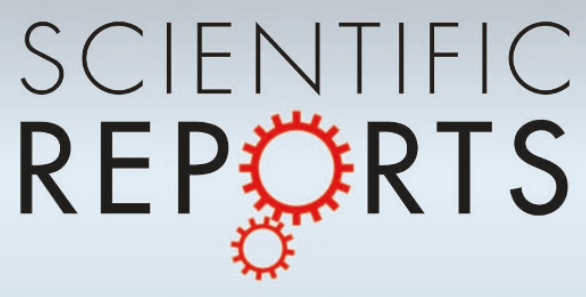

OPEN

SUBJECT AREAS:

METALS AND ALLOYS

MECHANICAL PROPERTIES

STRUCTURAL PROPERTIES

COMPUTATIONAL METHODS

Received

28 March 2013

Accepted

11 June 2013

Published

8 July 2013

Correspondence and requests for materials should be addressed to

Y.D.W. (ydwang@ mail.neu.edu.cn);

Y.L.H. (ylhao@imr.ac. $\mathrm{cn}) ;$ Y.W. (wang.

363@osu.edu) or R.Y.

(ryang@imr.ac.cn)

\section{New intrinsic mechanism on gum-like superelasticity of multifunctional alloys}

\author{
Jia-Peng Liu ${ }^{1,2}$, Yan-Dong Wang ${ }^{1}$, Yu-Lin Hao ${ }^{3}$, Yunzhi Wang ${ }^{4,5}$, Zhi-Hua Nie' ${ }^{2}$, Dong Wang ${ }^{4,5}$, Yang Ren ${ }^{6}$,
} Zhao-Ping Lu', Jinguo Wang ${ }^{7}$, Haoliang Wang ${ }^{3}$, Xidong Hui ${ }^{1}$, Ning Lu ${ }^{7}$, Moon J. Kim ${ }^{7} \&$ Rui Yang $^{3}$

\begin{abstract}
'State Key Laboratory for Advanced Metals and Materials, University of Science and Technology, Beijing 100083, P. R. China, ${ }^{2}$ School of Materials Science and Engineering, Beijing Institute of Technology, Beijing 100081, P. R. China, ${ }^{3}$ Shenyang National Laboratory for Materials Science, Institute of Metal Research, Chinese Academy of Sciences, Shenyang 110016, P. R. China, ${ }^{4}$ Department of Materials Science and Engineering, The Ohio State University, Columbus, Ohio 432 10, USA, ${ }^{5}$ Frontier Institute of Science and Technology, State Key Laboratory for Mechanical Behavior of Materials, Xi'an Jiaotong University, Xi'an 710049, China, ${ }^{6}$ X-ray Science Division, Argonne National Laboratory, Argonne, IL 60439, USA, ${ }^{7}$ Department of Materials Science and Engineering, The University of Texas at Dallas, Richardson, TX 75080, USA.
\end{abstract}

Ti-Nb-based Gum Metals exhibit extraordinary superelasticity with ultralow elastic modulus, superior strength and ductility, and a peculiar dislocation-free deformation behavior, most of which challenge existing theories of crystal strength. Additionally, this kind of alloys actually displays even more anomalous mechanical properties, such as the non-linear superelastic behavior, accompanied by a pronounced tension-to-compression asymmetry, and large ductility with a low Poisson's ratio. Two main contradictory arguments exist concerning the deformation mechanisms of those alloys, i.e., formation of reversible nanodisturbance and reversible martensitic transformation. Herein we used the in-situ synchrotron high-energy $\mathrm{X}$-ray scattering technique to reveal the novel intrinsic physical origin of all anomalous mechanical properties of the Ti-24Nb-4Zr-8Sn-0.10O alloy, a typical gum-like metal. Our experiments provide direct evidence on two different kinds of interesting, stress-induced, reversible nanoscale martensitic transitions, i.e., the austenitic regions with B2 structure transform to $\alpha^{\prime \prime}$ martensite and those with BCC structure transform to $\delta$ martensite.

T he strong, but less stiffer, metallic materials that exhibit high strength and low elastic modulus have long challenged the intrinsic physical nature of metallic bonds. The crystal strength is determined by a critical shear stress to actuate the glide of crystallographic line-defects called dislocations, which is in proportion to the shear elastic modulus, $\mu$, i.e., approximately reaching $1 / 30$ of $\mu^{1}$. For metallic alloys with a Poisson's ratio, $v$, varying within a narrow range from 0.25 to $0.35^{2}$, the Young's modulus, E, is in linear relationship with the shear modulus via a simple relation $\mathrm{E}=2(1+v) \mu$. This physical metallurgical principle was broken by a recent discovery on a series of multicomponent body-centered-cubic (BCC) solid solutions based on Ti-Nb binary alloys termed as gum metals (GMs) that have extraordinary multifunctional properties such as ultralow elastic modulus, ultrahigh strength, and super-elasticity and excellent plasticity ${ }^{3}$. Experimental studies found no phase transformations, no high density of dislocations (even after $90 \%$ cold work), and no twinning, leading to a hypothesis of dislocation-free deformation via the growth of nanodisturbances ${ }^{4}$ to form giant faults ${ }^{5,6}$. The activation of such mechanism was attributed to the combination of low ideal shear strength, induced by instability of the parent $\beta$ phase ${ }^{7}$, and high critical shear stress required by conventional dislocation glide ${ }^{3,6}$. However, this hypothesis has been challenged by the well-known conventional deformation mechanisms, such as dislocation glide $^{8}$, deformation twinning ${ }^{8,9}$, and stress-induced martensitic transformation from $\beta$ (BCC) austenite to $\alpha^{\prime \prime}$ (orthorhombic) martensite ${ }^{9-11}$, with essential differences from the nanodisturbance hypothesis in which the socalled 'non-crystallographic' partial dislocations with the Burgers vectors having non-quantized magnitude exist and play a crucial role. Therefore, the underlying deformation mechanism responsible for the peculiar properties of this new class of metallic materials still remains elusive.

Here we use synchrotron-based high-energy X-ray diffuse scattering (HE-XRDS), in combination with computer simulations, to explore in-situ the deformation behavior of a gum-type Ti-24Nb-4Zr- $8 \mathrm{Sn}-0.10 \mathrm{O}$ (in weight percent) alloy (abbreviated as Ti2448 from its chemical composition) ${ }^{12}$. Besides the high penetration, low absorption, great access to the reciprocal space, and high reciprocal resolution, the HE-XRDS technique can trace in-situ small changes in crystal structure, size, and elastic strain state even for nanocrystalline domains with a small volume fraction during deformation and phase transformation in bulk samples ${ }^{13}$. It is established in our 
present investigations that the Ti2448 alloy consists of a nanoscale B2 clusters embedded in a BCC matrix that has a slightly different $\mathrm{Nb}$ content from that of the B2 regions. Furthermore, our experiments provide unambiguous evidence on the formation of two different kinds of stress-induced nano-scale martensites, $\alpha^{\prime \prime}$ and $\delta$, originating from the B2 nanoclusters and the BCC matrix, respectively. The nano-scale martensites consist of frustrated nanodomains of individual martensitic variants, which are distinctively different from the normal self-accomodating polytwinned martensitic plates. We believe that stress-induced reversible transformations of B2 to $\alpha^{\prime \prime}$ and BCC to $\delta$ and switch of nanodomains of these martensites contribute to the anomalous mechanical behavior of the gum-like metals.

\section{Results}

Figure 1a gives the standard stress-strain (S-S) curves mechanically tested on single crystal Ti2448 loaded along the $[110]_{\beta}$ direction under both tension and compression ${ }^{12}$ at room temperature. Interestingly, the two-step superelasticity with a stress plateau at $\sim 240 \mathrm{MPa}$, accompanied by a jump in the strain of $\sim 2 \%$, is observed only under tension, in clear contrast to the almost linear S-S curve obtained under compression. Thus there is clearly a tension-compression asymmetry ${ }^{12}$. Moreover, the detailed changes in slopes of the S-S curves at low strain values (shown in the inset of Fig. 1a) indicate completely different non-linear elastic deformation behaviors under tension and compression. With increasing stress, an obvious reduction in modulus is observed under tension, but a slight increase in modulus is observed under compression.

The HE-XRDS reveals the existence of nano-sized domains of $\alpha^{\prime \prime}$ martensite ${ }^{14}$ prior to loading as evidenced from weak diffuse scattering signals at $\{021\}_{\alpha^{\prime \prime}}$ (indicated by the red circles) located at the middle of $\{110\}_{\beta}$ and $\{200\}_{\beta}$ diffraction spots (Fig. 1b). The nearly equal intensity of $\{021\}_{\alpha^{\prime \prime}}$ spots in the reciprocal space with cubic symmetry suggests no variant selection among the $\alpha$ " nano-sized martensitic domains. More importantly, the in-situ HE-XRDS experiments verify through distinctive changes in the scattering signals during tensile and compressive loading that the superelastic behavior of the alloy involves complex phase transformation processes. Under tension the stress-induced phase transformation from B2 nanoclusters to $\alpha$ " is evidenced by the obvious increase in the $(021)_{\alpha^{\prime \prime}}$ diffuse scattering signals (Fig. 1c), while the same phase transformation is suppressed under compression (as evidenced by the vanishing of the $(021)_{\alpha^{\prime \prime}}$ diffuse scattering signals) and, instead, $\omega$ phase starts to form at a compressive stress of $\sim 500 \mathrm{MPa}$ (as indicated by the HE-XRDS pattern displayed in the inset of Fig. 1a). After the applied compressive stress is removed, the $\omega$ phase remains (as indicated by the HE-XRDS patterns provided in the Supplementary Fig. S5 and Movie S1).

The crystal structure of $\alpha^{\prime \prime}$ is determined by using high-energy $\mathrm{X}$ ray diffraction $(\mathrm{HE}-\mathrm{XRD})$ by rotating the sample around $[110]_{\beta}$. It is
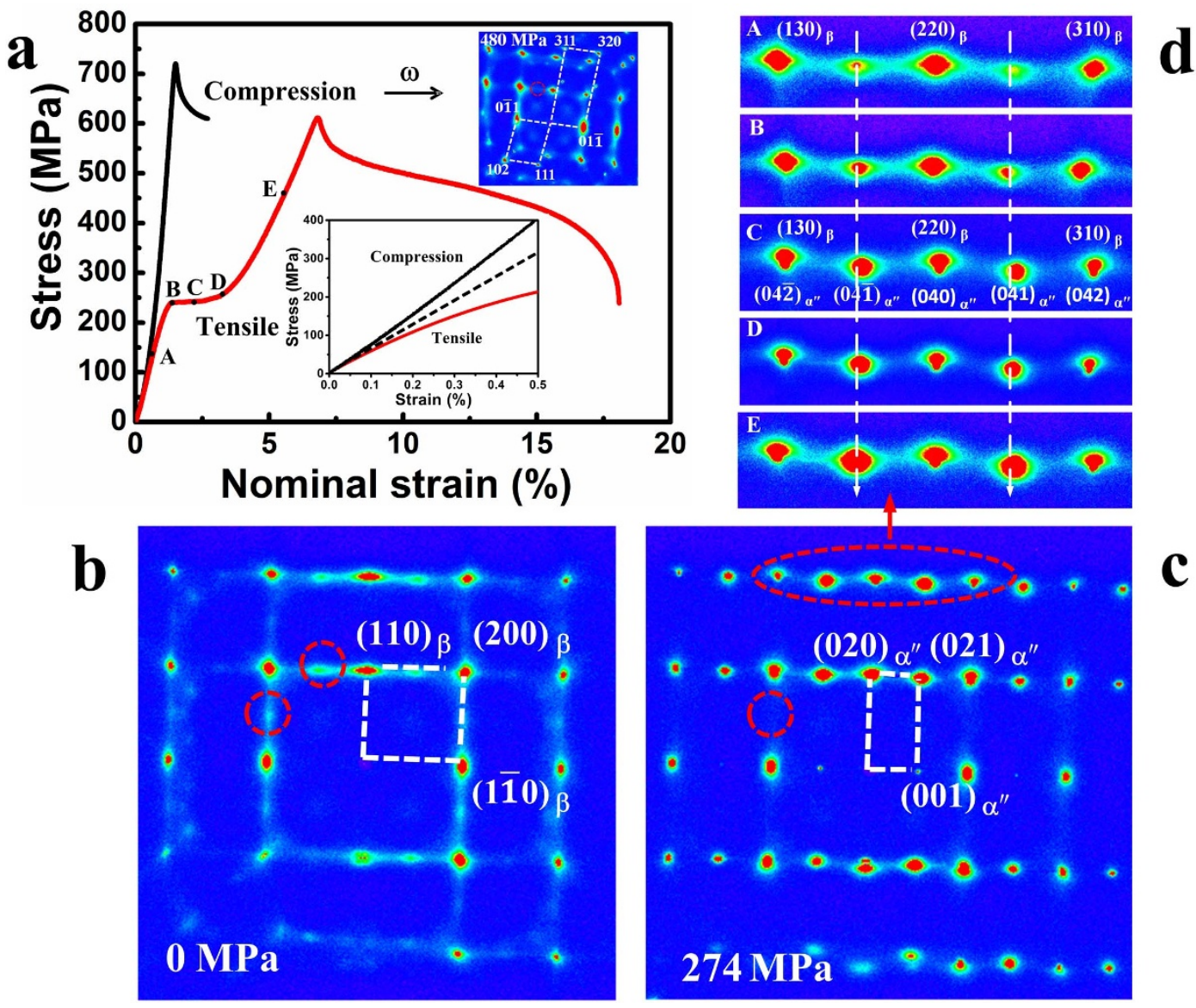

Figure 1 | High-energy X-ray diffuse scattering (HE-XRDS) studies of deformation behaviour for gum-type Ti-24Nb-4Zr-8Sn-0.10O single crystal loaded along [110] orientation. (a), Stress-strain (SS) curve for single crystal under tensile and compression modes. (b), (c), The HE-XRDS pattern for the single crystal under tensile loading at $0 \mathrm{MPa}(\mathrm{b})$ and $274 \mathrm{MPa}$ (c) with the X-ray incident beam along $[001]_{\beta}$ direction. Note that the existence of weak diffuse scattering signals located at the $(02 \overline{1})_{\alpha^{\prime \prime}}$ position is highlighted with red dotted circle in (b). (d), The detailed change in scattering patterns as highlighted in the red dotted circle in (c) at different stresses marked with the same symbols shown in (a). The insets in (a) are the scattering pattern under compression loading and enlarged S-S curve at a low strain, respectively, showing the diverse elastic modulus at different stress states. All newly-emerged diffraction spots appearing under compression mode can be indexed as $\omega$ phase. 
a C222 orthorhombic structure (space group No. 21) with lattice parameters of $a=3.222 \AA, b=4.788 \AA$ and $c=4.667 \AA$. It is an ordered structure and the corresponding disordered structure has a space group Cmcm (No. 63). Most of the diffraction spots of $\alpha$ " overlap with those of the $\beta$ phase, indicating a good lattice match between $\alpha^{\prime \prime}$ and $\beta$ at the given orientation relationship, i.e., $(110)_{\beta} / /(010)_{\alpha^{\prime \prime}}$ and $[001]_{\beta} / /[100]_{\alpha^{\prime \prime}}$. Some superlattice diffraction spots, such as $(021)_{\alpha^{\prime \prime}}$ and $(041)_{\alpha^{\prime \prime}}$, are from the $\alpha^{\prime \prime}$ phase due to both atomic occupation order and lattice modulation. The lattice modulation could be caused by the $\{110\}_{\mathrm{B} 2}<1 \overline{1} 0>_{\mathrm{B} 2}$ lattice shuffle involved in the stress-induced phase transformation from B2 to $\alpha$ " through the phonon softening mechanism. Due to an obvious difference in elastic modulus between $\alpha^{\prime \prime}$ and $\beta$ as seen from their different diffraction elastic moduli calculated from the slop of their respective lattice strains (see the Suplimentary Information (SI)), even those fundamental overlapping lattice peaks finally separated under a tensile stress. The detailed phase transformation path during tensile loading can be seen clearly from the increase in the $(041)_{\alpha^{\prime \prime}}$ scattering signals at different stresses (Fig. 1d), as indicated by the different letters marked on the S-S curve in Fig. 1a. It should be noted that, with increasing tensile stress, the dspacing of $\alpha^{\prime}$ along the loading direction becomes larger than that of $\beta$ as indicated by a rapid shift in the peak position belonging to $(041)_{\alpha^{\prime \prime}}$ scattering toward the beam center. Finally, the $(220)_{\beta}$ diffraction peak splits into two peaks, i.e., $(220)_{\beta}$ and $(040)_{\alpha^{\prime \prime}}$ (see the
Supplementary Movies S2 and S3), which suggests that the $\alpha$ " phase is much less stiffer than the $\beta$ phase. After the applied stress was removed, all the diffuse scattering spots returned to the initial state, suggesting that the phase transformation is reversible in contrast to the irreversible phase transformation under compression along $[110]_{\beta}$.

The detailed analyses of the HE-XRDS data offer rich information on the phase transformation kinetics (PTK), the change in domain size (DS), and the local lattice strain of martensitic domains, for perceiving the microscopic deformation mechanisms in this interesting alloy system. Fig. 2a shows the variation of volume fraction (VF) of $\alpha^{\prime \prime}$ as a function of applied stress, $\sigma$, which is obtained from the change in the diffuse scattering integral intensity for its $(021)_{\alpha^{\prime \prime}}$ peak using a proper scale method (described in the SI). Fig. 2b shows the corresponding change in DS of $\alpha$ " derived from the full-width at half maximum (FWHM), as demonstrated in the inset of Fig. $2 b$. Based on the PTK for $\alpha$ ", three different transformation regions are defined: (I) a slight increase in VF, accompanied by a slight increase in DS (from $\sim 1.5$ to $\sim 2.2 \mathrm{~nm}$ ) (noted as O-A-B); (II) a quick increase in VF, accompanied by an initially rapid increase in DS from $\sim 2.2$ to $\sim 8 \mathrm{~nm}$ that remained constant thereafter (noted as B-C-D); and (III) a slow increase in VF with a constant DS (noted as D-E). It should be noted that there is a slight shift in the stress level between the macroscopic S-S curve (Fig. 1a) and the PTK curve (Fig. 2), which $\mathbf{a}$
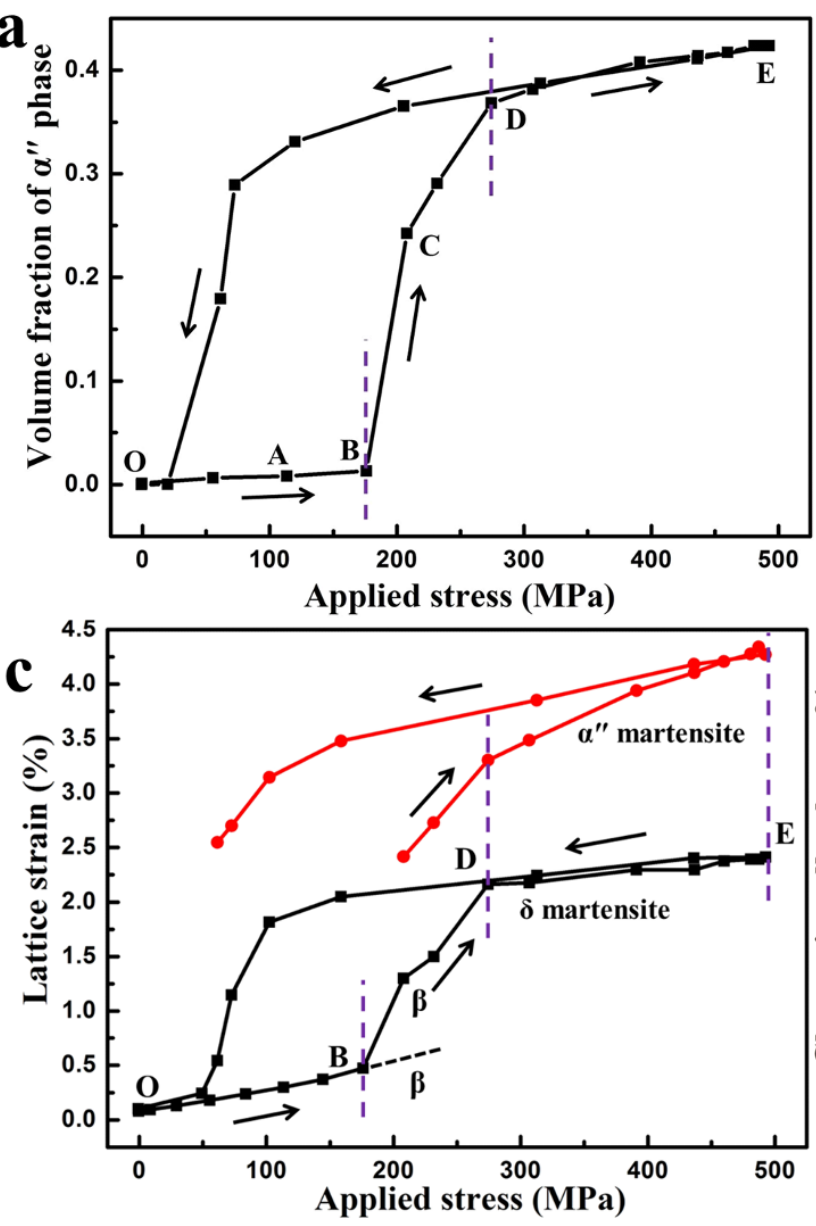
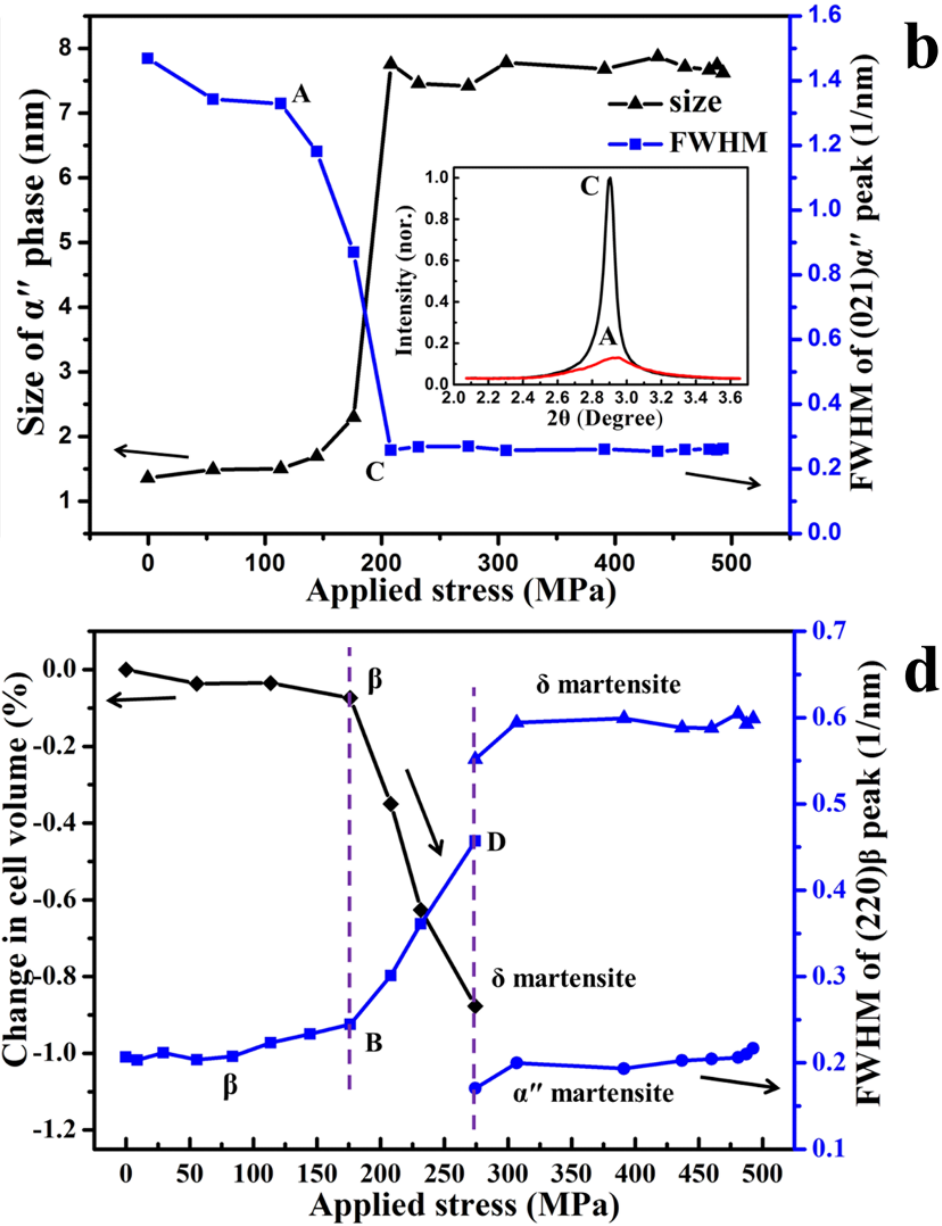

Figure $2 \mid$ High-energy X-ray diffuse scattering (HE-XRDS) studies of phase transition kinetics and microstructural features for different phases for gum-type Ti-24Nb-4Zr-8Sn-0.10O single crystal. (a), Volume fraction of ordered $\alpha$ " phase as a function of applied stress. (b), The domain size and full-width at half maximum (FWHM) for $(02 \overline{1})_{\alpha^{\prime \prime}}$ scattering peak of frustrated $\alpha^{\prime \prime}$ martensite as a function of applied stress. (c), The change in lattice strain obtained from $(220)_{\beta}$ for $\beta$ phase and frustrated $\delta$ martensite, and $(040)_{\alpha^{\prime \prime}}$ for $\alpha^{\prime \prime}$ martensite as a function of applied stress. (d), The FWHM for $\beta, \delta$, and $\alpha^{\prime \prime}$ phases as a function of applied stress. The inset in $(b)$ is the scattering patterns of $(02 \overline{1})_{\alpha^{\prime \prime}}$ at different transformation stages. Note the change of cell volume from $\beta$ to frustrated $\delta$ martensite under a tensile loading stress in (d). 
is due mainly to the different strain rates used in the two studies (see supplementary information). The most pronounced change occurred in the transformation regions from B-D.

The almost constant value in FWHM for the $(021)_{\alpha^{\prime \prime}}$ peak (shown in Fig. 2b) after entering into the transformation region (C-D-E) provides a convenient way to separate the lattice strain and FWHM for $\alpha$ " and $\beta$ from the overlapped diffraction peaks (as shown respectively in Figs. $2 c$ and $2 \mathrm{~d}$ ). The slope of the lattice strain vs. applied stress curve remains almost constant at low stresses $(\mathrm{O}-\mathrm{B}$ in Fig. 2a), from which a diffraction elastic modulus of $\sim 58 \mathrm{GPa}$ was estimated in the initial stage of elastic deformation and $\sim 30 \mathrm{GPa}$ when the applied stress reaches $\sim 180 \mathrm{MPa}$. Those values are in good agreement with the average Young's modulus measured by the mechanical method.

The lattice expansion caused by the phase transformation from $\beta$ to $\alpha$ " can be evidenced from the sudden jump in the lattice strain curve of $\alpha^{\prime \prime}$ (Fig. 2c), which should not be surprising due to completely different crystal structures of $\alpha$ " and $\beta$. The astonishing finding is the existence of a sudden jump in both lattice strain and FWHM for $\beta$, corresponding exactly to the transformation from $\beta$ into $\alpha$ ". This indicates that, besides the stress-induced $\alpha$ " phase, there is another specific phase transformation from $\beta$ to a new phase. Our dedicated HE-XRD experiment verified that the new phase has an orthorhombic structure, with lattice parameters of $a=3.190 \AA, b=4.762 \AA$, and $c=4.680 \AA$. Although this structure is close to that of the $\alpha$ " phase with similar lattice parameters, it belongs to a different space group (with the symbol $\mathrm{cmcm}$, No. 63) (see the detailed change of cell parameters under loading in the supplementary information). We refer to this new phase as $\delta$ hereafter. The large value of FWHM and abrupt decrease in the unit cell volume $(\sim-0.9 \%)$ as shown in Fig. $2 \mathrm{~d}$ after entering the region $\mathrm{D}-\mathrm{E}$ indicate that the remaining matrix has been transformed into nanodomains of individual variants of the $\delta$ martensite. The main difference between the $\delta$-phase and the $\alpha$ "-phase is that the former is a disordered structure without lattice modulation, while the latter is an ordered structure with a shuffle of adjacent $\{110\}_{\mathrm{B} 2}$ planes, which could originate from different regions (e.g., nano domains of $\mathrm{B} 2$ clusters embeded in the BCC matrix, with slightly different compositions as well, as will be discussed below) in the initially inhomogeneous microstructure formed during the sample fabrication. The large FWHM value obtained for the $\delta$ martensite indicates that its domain size is rather small. Based on the measured FWHM, the effective size, $\mathrm{Dv}=3.4 \mathrm{~nm}$, can be estimated from the Debye-Scherrer formula, $\mathrm{Dv}=\lambda /(\mathrm{H} \cdot \cos \theta)$ (where $\lambda$ is the wavelength of the radiation and $H$ is the integral breadth of a reflection located at $2 \theta)^{15}$. When the applied stress is further increased (D-E in Fig. 2a), both lattice strain and FWHM of the $\delta$-phase remain almost constant.

Fig. $3 \mathrm{a}$ is the high-angle annular dark-field (HAADF)-scanning transmission electron microscopy (STEM) image with the incident electron beam along the $[001]_{\beta}$ zone axis of the single crystal sample. The image (usually called as the Z-contrast image), formed only by very high-angle, incoherently-scattered electrons, as opposed to Bragg-scattered electrons, is highly sensitive to variations in the atomic number of elements contained in the sample. Heavier atoms show higher contrast, while lighter atoms appear darker in the image. The existence of heavy-atom ( $\mathrm{Nb}, \mathrm{Zr}$, or $\mathrm{Sn}$ )-rich and -lean regions can be revealed by adjusting the contrast of the HAADF-STEM image (Fig. 3b). The existence of two kinds of $\mathrm{Nb}$-rich and Ti-rich $\mathbf{a}$
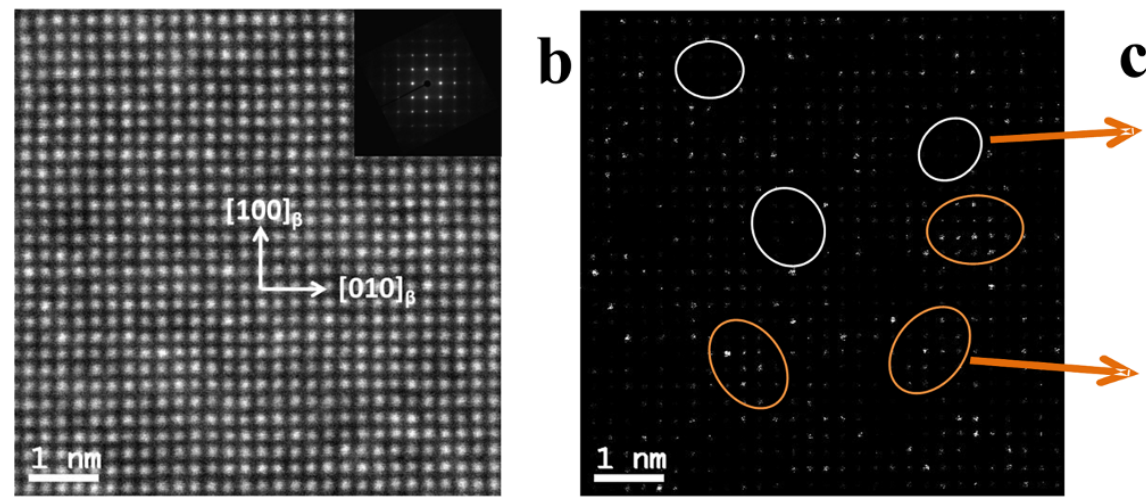

Q
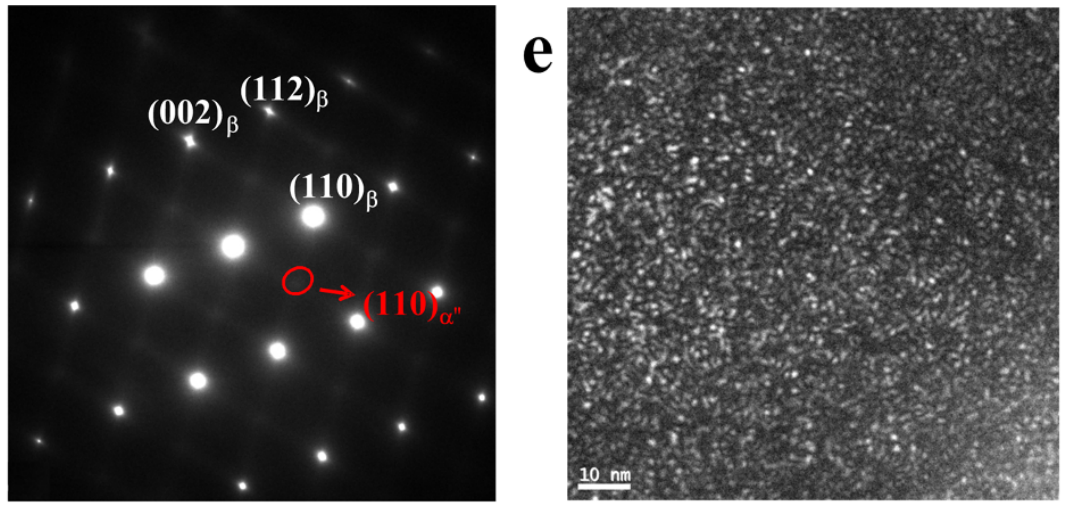
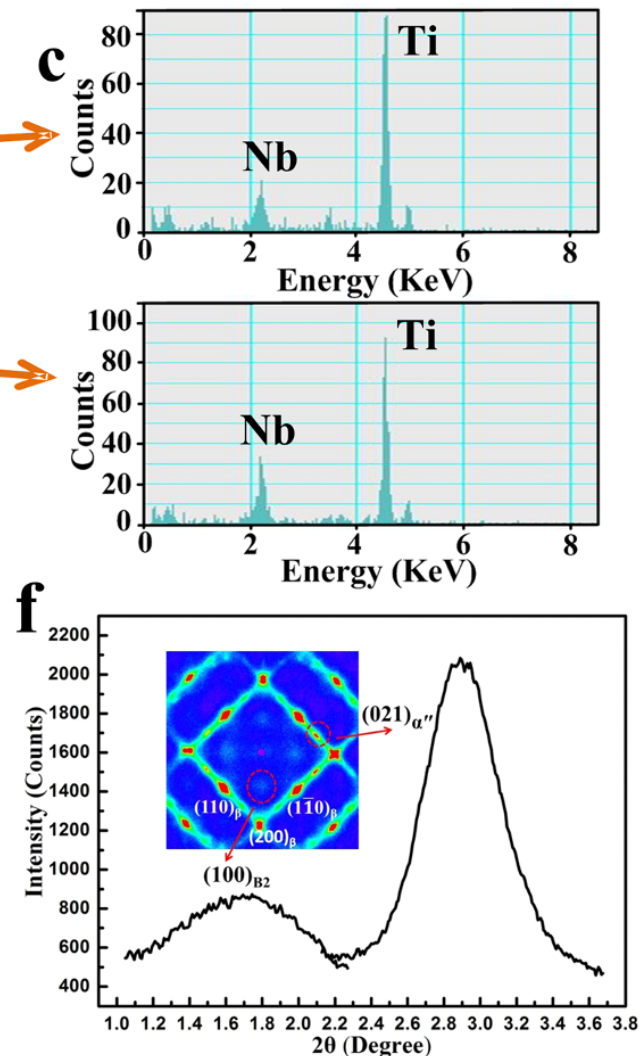

Figure $3 \mid$ The TEM observations to show the origin of the different phase transitions. (a), (b), and (c) The HAADF-STEM image for [001 $]_{\beta}$ crystal axis with differently adjusted contrasts to show the existence of clusters of heavy atoms in the B2-ordered structure with the EDS spectra (c) at the $\mathrm{Ti}$ - and $\mathrm{Nb}$ - rich area marked inside those white and purple frames in (b). (d), (e), The nano-beam electron diffraction pattern to show the existence of weak diffuse scattering at $1 / 2(112)_{\beta}$ position and its corresponding dark field image to show the random distribution of $\alpha^{\prime \prime}$ nanodomains and B2 clusters inside the disordered BCC matrix. (f), The 1-dimensional HE-XRDS pattern to show the strong diffuse scattering signals located at $(001)_{\beta}$ and $(021)_{\alpha^{\prime \prime}}$ positions. 
phase-separated domains is further confirmed by the spectra of energy-dispersive X-ray spectroscopy (EDS) (Fig. 3c), which show $\mathrm{Nb}$-rich and $\mathrm{Nb}$-lean regions. The existence of $\mathrm{B} 2$ clusters can be identified from the STEM image in the heavy-atom-rich areas (shown in Fig. 3b). The selected area electron diffraction pattern for the Ti2448 alloy along the $[001]_{\beta}$ zone axis does not show an obvious diffuse scattering signal (see the inset of Fig. 3a), which is in contrast to that observed in the Ti-23Nb-1O alloy ${ }^{16}$. Different from what was reported in $\mathrm{Ti}-23 \mathrm{Nb}-1 \mathrm{O}$ alloy, our observation is due mainly to the small amount of oxygen and confirms that almost no modulated structure in B2 nanodomains was formed in the initial state before loading. The nano-beam electron diffraction pattern along the $[011]_{\beta}$ zone axis indeed shows the existence of weak diffuse scattering located at $1 / 2(112)_{\beta}$ (in coincidence with $\left.(021)_{\alpha^{\prime \prime}}\right)$ position for Ti2448 (Fig. 3d), while its corresponding dark field image shows the random distribution of $\alpha^{\prime \prime}$ nanodomains and B2 clusters in the initial sample (Fig. 3e). The existence of the B2-clusters and $\alpha$ " nanodomains is supported strongly by the HE-XRDS signals located at the $(001)_{\beta}$ (characterized with B2 structure) and $(021)_{\alpha^{\prime \prime}}$ peak positions for the undeformed Ti2448 alloy (shown in Fig. 3f).

\section{Discussion}

The phase transformation described above for the Ti2448 alloy is completely different from that of normal martensitic transformation (NMT). Based on the TEM observations, the sample before loading is non-uniform in both structure and composition and consists of three regions: $\mathrm{Nb}$-rich $\mathrm{B} 2$ clusters (austenite), $\mathrm{Nb}$-rich $\alpha$ " static nano-scale domains (could also be called as embryos because they grow only under load and return to their original states when the load is removed) and Nb-lean BCC regions, as schematically shown in Fig. 4a. Upon loading, the existing $\alpha$ " embryos grow and the B2 and BCC regions transformed to nanodomains of $\alpha^{\prime \prime}$ and $\delta$ martensites, respectively, as schematically shown in Fig. $4 \mathrm{~b}$. Both the number and size of the nanodomains of these two kinds of martensites increase under the applied load, leading to the observed superelasticity and nonlinear elastic behavior of the system. But their growth is confined by chemical and structural non-uniformities in the austenite and by each other as neighboring domains. As no martensitic domains of any other variants are found in the whole HE-XRDS patterns, all domains belong to the same variant of martensite that is favored by the tensile load.

The stress-induced martensites in our sample are different from the polytwinned, long-range ordered strain state consisting of multiple self-accommodating variants as those observed during NMT. The long-range ordered strain state is suppressed by the random spatial distribution of various chemically and structurally different nanodomains in the alloy (see, e.g., Fig. 3e) that transform into different martenstic phases upon loading. Also note that the chemical and structural (degree of B2 ordering) non-uniformities present in the parent austenite phase, whose homogenization requires atomic diffusion, are preserved in the stress-induced martensitic phases.

The fine-scale chemical and structural non-uniformities (i.e., ordered B2 clusters in the disordered BCC matrix) in the austenite observed in the experiment could be produced by spinodal decomposition followed by $\mathrm{B} 2$ ordering in the Nb-rich domains ${ }^{17}$ or by the pseudo-spinodal decomposition mechanism ${ }^{18,19}$ in which thermal fluctuations bring concentrations of local regions beyond a critical concentration, $\mathrm{c}_{0}$, at which both the ordered and disordered phases have the same free energy, resulting in congruent ordering that is followed by phase separation. The presence of the $\alpha$ " embryos could be attributed to the random substitutional alloying elements in the alloy such as $\mathrm{Nb}, \mathrm{Zr}$ and $\mathrm{Sn}$ whose atomic sizes are quite different from the host atoms (Ti). Both experimental and theoretical studies have shown that point defects could broaden martensitic transition temperature and reduce martensitic domain size $e^{20-22}$.

To understand the effect of these fine-scale chemical and structural non-uniformities in the austenite on MTs, we carry out computer simulations using the phase field method ${ }^{21,23,24}$ based on Landau theory. Note that our present simulations do not concern the kinetic processes that lead to the initial non-uniformities in the austenite. Instead we focus on the subsequent MTs under the influence of such non-uniformities in the parent phase. To do so we introduce two effects associated with these non-uniformities in our model, i.e., the local transition temperauture effect (LTTE) and local field effect (LFE) $)^{21,22}$. The LTTE allows us to have different stabilities

a
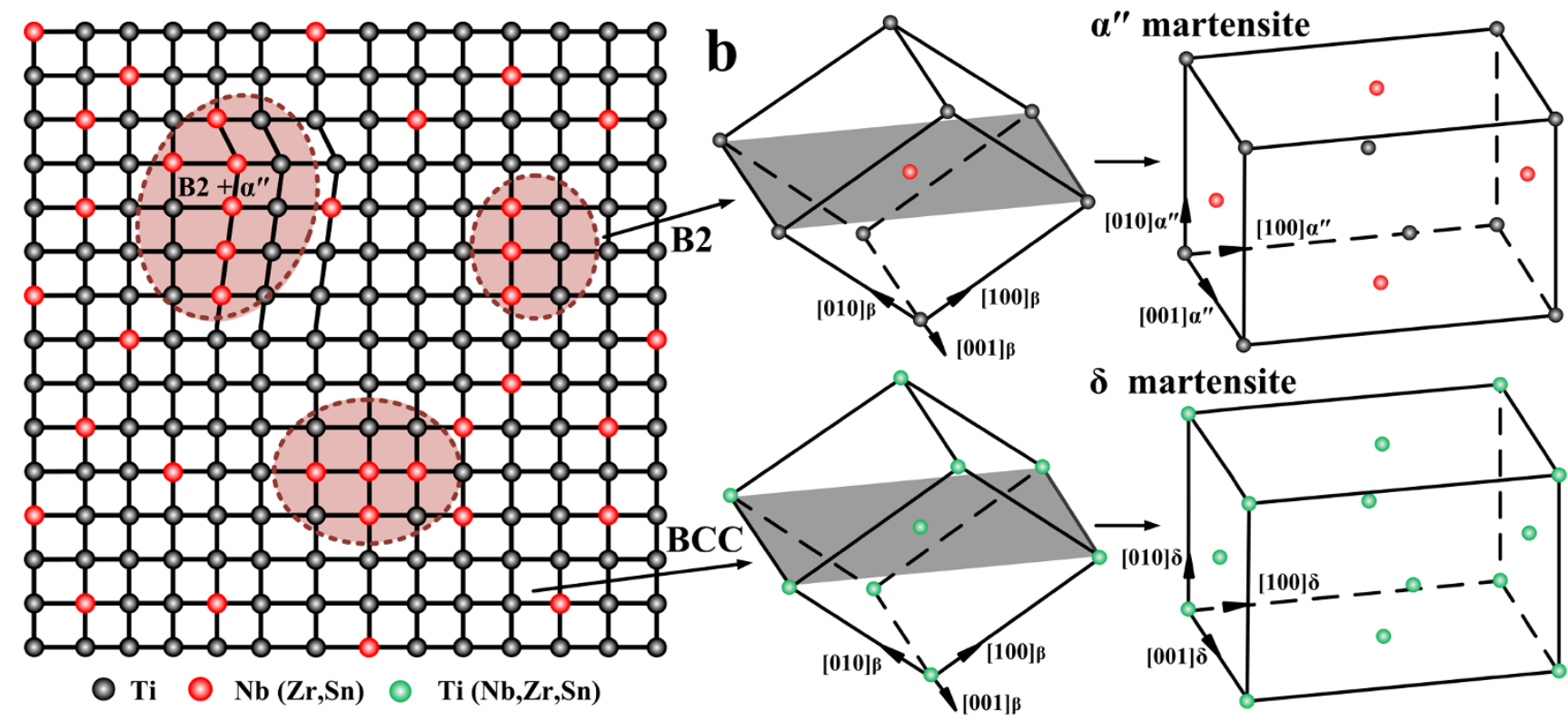

$\delta$ martensite

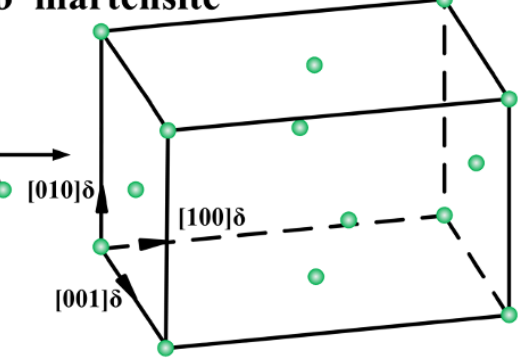

Figure 4 Schematics to show the dual martensite phase-transformation mechanism during deformation for gum-type Ti-24Nb-4Zr-8Sn-0.10O alloy. (a), The schematic diagram to show the initial microstructure that consists of three parts, i.e., Nb-rich B2 regions (austenite), Nb-rich $\alpha$ " martensitic domains (embryos) and Nb-lean BCC regions. (b), two kinds of phase transition scenarios during tensile deformation, i.e., the formation of (ordered) frustrated $\alpha^{\prime \prime}$-martensite from B2-ordered structure and (disordered) frustrated $\delta$-martensite from BCC-disordered structure. 
(i.e., different martensitic start temperatures) for the two martensitic phases (i.e., $\alpha$ " in B2 regions and $\delta$ in BCC regions), while the LFE allows us to capture the effect of local lattice distortion caused by atomic size differences between the random substitional elements and the host atom mentioned above. In the model different LFEs are assumed for the BCC and B2 regions. Figure 5 shows the S-S curve and related properties of the Ti2448 alloy simulated by the phase field method. According to the experimental observations discussed above (Fig. 3b), the initial microstructure in our simulations (Fig. 5c) consists of Nb-rich (B2-structured) regions (light shade in Fig. 5c) with $\alpha^{\prime \prime}$ martensitic embryos and Nb-lean (BCC structure) regions (dark shades in Fig. 5c) with $\delta$ martensitic embryos. In our model, these martensitic embryos are induced by the random local fields associated with the substitutional elements. The B2 regions will transform into $\alpha$ " martensite while the BCC region will transform into the $\delta$ martensite.

Figure 5a shows the non-linear S-S curve under loading and unloading, with the inset showing the enlarged portion of S-S curve in the elastic region. Fig. 5b shows the calculated volume fraction change of martensitic domains in the BCC and B2 austenite matrix regions, respectively, as function of stress corresponding to the S-S curve shown in Fig. 5a. The volume fraction increases when the applied stress increases, resulting from the effect of stress-induced MT process. The stress-induced MT in both the BCC and B2 austenite occurs at a similar stress level (with different critical stress as shown in Fig. 5b). Fig. 5d shows the related microstructural evolution in the $\mathrm{BCC}$ and $\mathrm{B} 2$ austenitic regions, respectively, upon loading, where red and blue colors represent different martensitic variants (embryos of martensitic variants exist as shown by the light red or light blue regions) and green color represents the parent phase.

There are six martensitic variants for $\alpha^{\prime \prime}$ and $\delta$ respectively, and here we use the red color to describe the favorable martensitic variant under the applied stress and the blue color to describe the other unfavorable martensitic variants. When a load is applied, the system transforms gradually from a state consisting of nanodomains of all variants (red and blue in both BCC and B2 regions) at zero load into nanodomains dominated by one variant (i.e., the red one) that is preferred by the applied stress. The volume fraction curves (Fig. 5b) under loading also show the gradual increase of nanodomains of both $\alpha^{\prime \prime}$ and $\delta$ martensites. The gradual growth of existing domains, the newly-generated domains favored by the stress field, and the switch of unfavorable domains towards the favored ones upon the continued increase of the load are the intrinsic physical origins of both the non-linear stress-strain behavior in the elastic region and the pseudo-elastic behavior in the inelastic region of the stress-strain curve. Thus our computer simulations starting with nanodomains of BCC and B2 austinitic regions have shown the existence of two different stress-induced martensitic transformations and reproduced the deformation behavior of the Ti2448 alloy without considering any dislocation-related plastic deformations.

In sumary, the in-situ synchrotron-based high-energy X-ray scattering experiments, in combination with TEM observations reveal for the first time the existence of nanoscale chemical and structural nonuniformities and stress-induced dual martensitic transformations in Ti2448 GMs. The phase field simulations using the phase field method that takes into account of the nanoscale non-uniformities
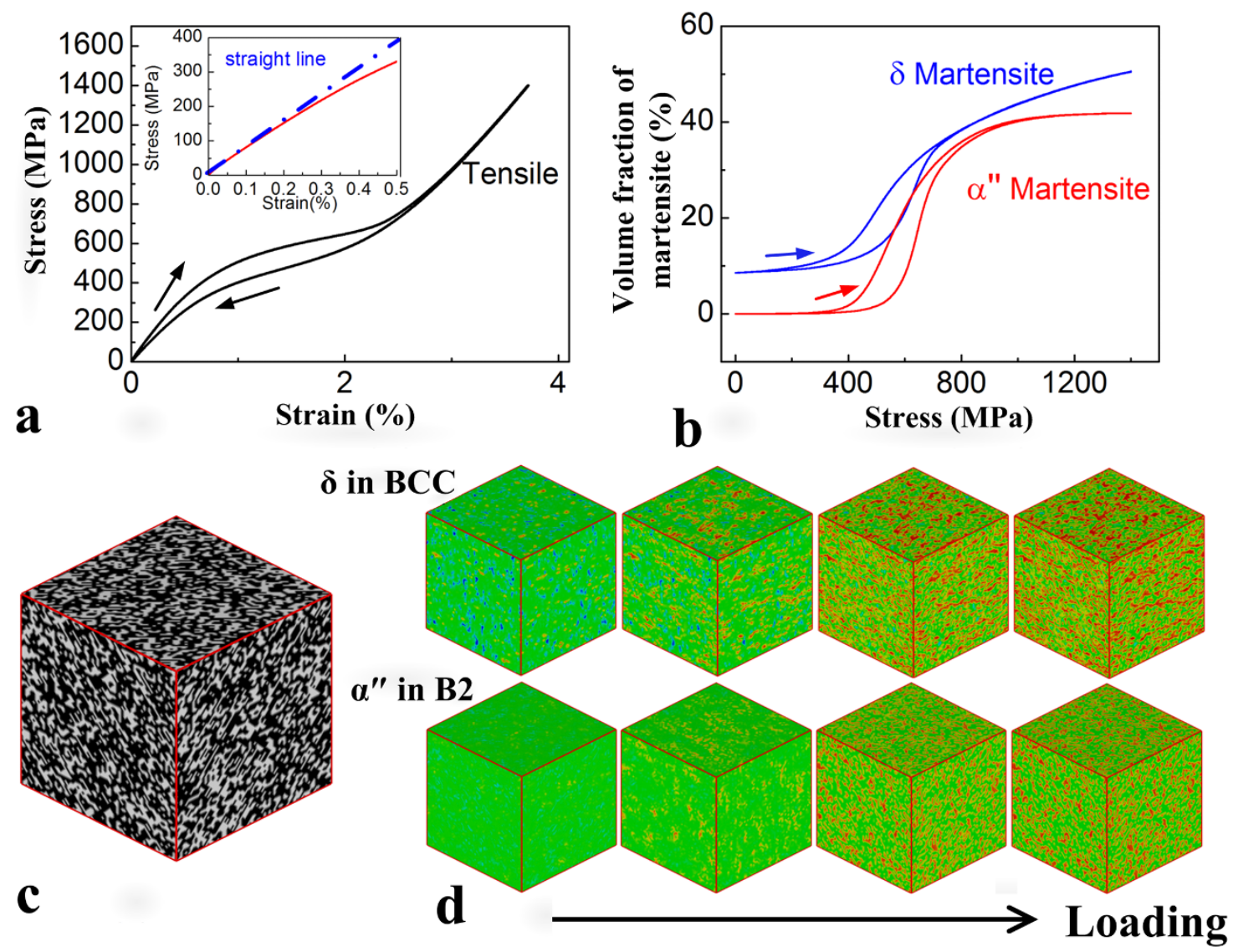

Figure $5 \mid$ Phase field simulations on the deformation behaviour for gum-type Ti-24Nb-4Zr-8Sn-0.10O single crystal under tensile loading along [110] orientation. (a), Calculated tensile stress-strain curves along [110] direction, with the inset describing the enlarged S-S curves in elastic part. (b), Related martensitic volume fraction in disordered BCC regime and ordered B2 regime. (c), Ordered B2 structure (white color) and disordered BCC structure (black color) in our system. (d), Evolution of nano-sized martensitic domains in the ordered B2 regime and disordered BCC regime, respectively, under loading. There the green color describes the parent phase, red and blue colors describe the martensitic variants. 
in austenite as well as possible effect from atomic size misfitting of the substitutional elements reproduce the deformation behavior of the alloy observed in the experiments. Thus the current study provides new insight into the intrinsic deformation mechanism for the anomalous mechanical behaviors of the gum-type metallic materials. The understanding of the physical origins of the stress-state dependence of elastic modulus during elastic deformation indeed benfits greatly designing new advanced functional and structural materials with wide applications in the whole materials spectrum.

\section{Methods}

Sample preparation. Master ingots with a composition of Ti-24Nb-4Zr-8Sn (mass\%) were made by arc melting under argon protection and hot-forged at 1273 and $1123 \mathrm{~K}$ into bars $25 \mathrm{~mm}$ in diameter. Single crystals were grown from the as-forged bars using an optical floating-zone furnace (FZ-T-12000-X-VP-S, Crystal System Inc.) at a crystal growth rate of $5 \mathrm{~mm} \mathrm{~h}^{-1}$ under a high-purity argon gas flow. The seed crystals were cut along the $\langle 100\rangle,\langle 110\rangle$ and $\langle 111\rangle$ directions after their orientations were determined by both X-ray diffraction (XRD) and Laue X-ray back reflection analyses within 1 degree of the desired orientation and then used to grow singlecrystal rods with the above orientations ${ }^{12}$. The dog-bone-shaped tensile and cubeshaped samples are cut from the grown single crystal for in-situ loading tension and compression tests, respectively.

High-energy X-ray scattering and transmission electron microscope (TEM). The in-situ X-ray diffuse scattering experiments were performed at the ID-11-C beam-line at the Advanced Photon Source (APS), Argonne National Laboratory (ANL). A monochromator with a $\mathrm{Si}$ (113) single crystal was used to provide an X-ray beam with the energy of $115 \mathrm{keV}$ and a loading frame with a displacement control was applied to the beam-line for studying the change in crystallographic structure during tensile and compressive loading. The size of the X-ray beam was $0.5 \times 0.5 \mathrm{~mm}$. The transmission geometry in a manner similar to TEM was used to monitor the change in crystallographic structures during loading. A two-dimensional detector (Perkin Elmer amorphous silicon) was employed for collecting the scattering patterns over a plane, encompassing the transverse direction and (nearly) the loading direction of the single crystal, with an exposure time matching a simultaneous rocking of the specimen within $\pm 5^{\circ}$ along the loading or transverse rotation axis for covering a large reciprocal space. Electron diffraction, dark field transmission electron microscopy (TEM), HR (high resolution)-TEM, HAADF-STEM and energy-dispersive X-ray (EDX) analyses were performed by using a JEOL ARM 200F microscope (JEOL, Tokyo, Japan) equipped with a Cs corrector, which was operated at $200 \mathrm{kV}$.

Phase field simulation. The model system considered is a single crystal undergoing an austenite (BCC $\beta$ phase) $\rightarrow$ martensite (orthorhombic phase) transformation ${ }^{21,24}$. The $\beta$ phase is separated to $42 \%$ (volume fraction) Nb-rich ordered B2 structure and $58 \% \mathrm{Nb}$-lean $\mathrm{BCC}$ structure. $\mathrm{B} 2$ region and $\mathrm{BCC}$ region will transform respectively into $\alpha^{\prime \prime}$ and $\delta$ martensitic phases, and the two martensitic phases have the same orthorhombic structure (See SI). To simplify the simulations, we assume that the $\mathrm{B} 2 \rightarrow \alpha^{\prime \prime}$ and $\mathrm{BCC} \rightarrow \delta$ transformations have the same transformation strain. We assume that the existence of alloying elements, such as $\mathrm{Zr}$ and $\mathrm{Sn}$, in Ti2448 produces randomly distributed lattice distortion (i.e., local field effect) according to the atomic radius difference among $\mathrm{Ti}(2.00), \mathrm{Nb}(2.08), \mathrm{Sn}(1.72)$ and $\mathrm{Zr}(2.16)$.

1. Kelly, A., Tyson, W. R. \& Cottrell, A. H. Ductile and brittle crystals. Philos. Mag. 15, 567-586 (1967).

2. Greaves, G. N., Greer, A. L., Lakes, R. S. \& Rouxel, T. Poisson's ratio and modern materials. Nature Mater. 10, 823-837 (2011).

3. Saito, T. et al. Multifunctional alloys obtained via a dislocation-free plastic deformation mechanism. Science 300, 464-467 (2003).

4. Gutkin, M. Y., Ishizaki, T., Kuramoto, S. \& Ovid'ko, I. A. Nanodisturbances in deformed gum metal. Acta Mater. 54, 2489-2499 (2006).

5. Ikehata, H. et al. First-principles calculations for development of low elastic modulus Ti alloys. Phys. Rev. B 70, 174113 (2004).

6. Yano, T., Murakami, Y., Shindo, D. \& Kuramoto, S. Study of the nanostructure of gum metal using energy-filtered transmission electron microscopy. Acta Mater. 57, 628-633 (2009).

7. Li, T., Morris, J. W., Jr., Nagasako, N., Kuramoto, S. \& Chrzan, D. "Ideal" engineering alloys. Phys. Rev. Lett. 98, 105503 (2007).
8. Yang, Y. et al. Evolution of deformation mechanisms of Ti-22.4Nb-0.73Ta-2Zr$1.34 \mathrm{O}$ alloy during straining. Acta Mater. 58, 2778-2787 (2010).

9. Yang, Y. et al. Stress-introduced $\alpha^{\prime \prime}$ martensite and twinning in a multifunctional titanium alloy. Scripta Mater. 58, 9-12 (2008).

10. Morris, J. W., Jr. et al. Anomalous transformation-induced deformation in $\langle 110\rangle$ textured gum Metal. Acta Mater. 58, 3271-3280 (2010).

11. Talling, R. J., Dashwood, R. J., Jackson, M. \& Dye, D. On the mechanism of superelasticity in Gum metal. Acta Mater. 57, 1188-1198 (2009).

12. Zhang, Y. W. et al. Elastic properties of Ti-24Nb-4Zr-8Sn single crystals with bcc crystal structure. Acta Mater. 59, 3081-3090 (2011).

13. Wang, Y. D. et al. Tracing memory in polycrystalline ferromagnetic shapememory alloy. Adv. Mater. 18, 2392-2396 (2006).

14. Obbard, E. G. et al. The effect of oxygen on $\alpha^{\prime \prime}$ martensite and superelasticity in Ti24Nb-4Zr-8Sn. Acta Mater. 59, 112-125 (2011).

15. Patterson, A. The Scherrer formula for X-ray particle size determination. Phys. Rev. 56, 978-982 (1939).

16. Tahara, M., Kim, H. Y., Inamura, T., Hosoda, H. \& Miyazaki, S. Lattice modulation and superelasticity in oxygen-added $\beta$-Ti alloys. Acta Mater. 59, 6208-6218 (2011).

17. Soffa, W. A. \& Laughlin, D. E. Recent experimental studies of continuous transformations in alloys: an overview. Proc. Int. Conf. solid to solid phase transformations. Met. Soc. AIME. 159-183 (1982)

18. Ni, Y. \& Khachaturyan, A. G. From chessbord-like tweed to chessboard nanowires during pseudo-spinodal decomposition. Nature Mater. 8, 410-414 (2009).

19. Rao, W. F. \& Khachaturyan, A. G. Superfunctionalities in nanodispersive precipitation-hardened alloys. Phys. Rev. Lett. 109, 115704 (2012).

20. Sarkar, S., Ren, X. \& Otsuka, K. Evidence for strain glass in the ferroelasticmartensitic system Ti50-xNi50+x. Phys. Rev. Lett. 95, 205702 (2005).

21. Wang, D., Wang, Y., Zhang, Z. \& Ren, X. Modeling abnormal strain states in ferroelastic systems: The role of point defects. Phys. Rev. Lett. 105, 205702 (2010).

22. Semenovskaya, S. \& Khachaturyan, A. G. Coherent structural transformations in random crystalline systems. Acta Mater. 45, 4367-4384 (1997).

23. Wang, Y. \& Khachaturyan, A. G. Three-dimensional field model and computer modeling of martensitic transformations. Acta Mater. 45, 759-773 (1997).

24. Khachaturyan, A. G. Theory of Structural Transformations in Solids (John Wiley \& Sons, New York, 1983).

\section{Acknowledgments}

This study was supported by the National Basic Research Program of China (973 Program) (Grant No.s 2012CB619405, 2012CB933901 and 2012CB619103), the National Natural Science Foundation of China (Grant No.s 51231002, 51071152 and 51271180), and the Fundamental Research Funds for the Central Universities (Grant No. 06111020). Use of the Advanced Photon Source was supported by the U.S. Department of Energy, Office of Science, Office of Basic Energy Science, under Contract No. DE-AC02-06CH11357. YW and DW thank the support of the U.S. Natural Science Foundation Grant No. DMR-1008349, National Basic Research Program of China (Grants No. 2012CB619402 and No. 2010CB631003) and National Natural Science Foundation of China (Grants No. 51201125).

\section{Author contributions}

Y.D.W., Y.L.H., Y.W., Z.P.L., R.Y. and Y.R. designed the research topic of this project. Y.L.H., H.W. and R.Y. prepared samples. Z.H.N., Y.R. and Y.D.W. performed synchrotron experiments. J.P.L., Y.D.W., Z.P.L. and X.H. analysed all synchrotron data. Y.W. and D.W. performed simulations. J.G.W., N.L. and M.J.K. performed T.E.M. study. Y.D.W., J.P.L., Y.W., Z.P.L., Y.L.H., D.W., Y.R., J.G.W. and R.Y. prepared the first version of manuscript and all authors took part in the discussions and paper writing.

\section{Additional information}

Supplementary information accompanies this paper at http://www.nature.com/ scientificreports

Competing financial interests: The authors declare no competing financial interests.

How to cite this article: Liu, J. et al. New intrinsic mechanism on gum-like superelasticity of multifunctional alloys. Sci. Rep. 3, 2156; DOI:10.1038/srep02156 (2013).

This work is licensed under a Creative Commons Attribution 3.0 Unported license. To view a copy of this license, visit http://creativecommons.org/licenses/by/3.0 